\title{
ARQUEOLOGIA PÚBLICA E ACERVO ARQUEOLÓGICO
}

Prof. Dr. Frederic Mario Caires Pouget ${ }^{1}$

Prof ${ }^{a}$. Drea . Aline Vieira de Carvalho

\section{RESUMO}

O texto visa estabelecer algumas relações entre aspectos teóricos e metodológicos da Arqueologia Pública com a gestão de acervos arqueológicos. Tendo como estudo de caso as práticas e políticas adotadas pelo Laboratório de Arqueologia Pública Paulo Duarte UNICAMP, o texto aborda os principais pontos conceituais da Arqueologia Pública que podem ser levados em consideração na formulação de políticas de gestão de acervos arqueológicos.

PALAVRAS-CHAVE: Arqueologia Pública, acervo, gestão, herança cultural.

\section{ABSTRACT}

The text aims to establish some relations between theoretical and methodological aspects of Public Archaeology with archaeological archive management. As it case study the practices and politics adopted by the Laboratório de Arqueologia Pública Paulo Duarte UNICAMP, the text approaches the main conceptual points of Public Archaeology that may be considered in the formulation of archaeological archive management politics.

KEYWORDS: Public Archaeology, archive, management, cultural heritage.

\section{INTRODUÇÃO}

\section{O LABORATÓRIO DE ARQUEOLOGIA PÚBLICA PAULO DUARTE}

\section{(LAP/NEPAM/UNICAMP)}

O LAP, atualmente localizado no Núcleo de Estudos e Pesquisas Ambientais (NEPAM/UNICAMP), insere-se nas pesquisas e discussões sobre arqueologia pública, ambiente e sociedade. Para a instituição, a relação entre a produção de conhecimento científico de forma simétrica (GONZALEZ-RUIBAL; HERNANDO, 2011) ${ }^{3}$ e significativa junto às comunidades interessadas é a principal ação política e de construção de caminhos de liberdade (FREIRE, 1999) ${ }^{4}$. A Arqueologia e, em especial, a produção da interpretação arqueológica é nosso principal caminho para debater temas próprios ao nosso cotidiano, entre eles, por exemplo, cultura material e poder. A diversidade de público é um ponto

\footnotetext{
${ }^{1}$ Pós-doc LAP/NEPAM/UNICAMP.

2 Professora e pesquisadora NEPAM/UNICAMP.

${ }^{3}$ GONZÁLEZ-RUIBAL, A.; HERNANDO, A.; POLITIS, G. Ontology of the self and material culture: arrow making among the Awáhunter-gatherers (Brazil). Journal of Anthropological Archaeology, n. 30, p. 1-16, 2011.

${ }^{4}$ FREIRE, Paulo. Educação como prática da liberdade. 23ạ ed. Rio de Janeiro: Paz e Terra, 1999.

\begin{tabular}{|l|l|l|l|l|l|l|}
\hline (C) Rev. Arqueologia Pública & Campinas, SP & v.11 & n.2 & p. 254 & Novembro/2017 & ISSN 2237-8294
\end{tabular}
} 


\section{ARTIGO}

\section{Revista de Arqueologia Pública}

central para a produção de reflexão dentro do LAP. Por conta disso, ao longo dos anos foram postos em marcha diversos projetos de atuação científica, como ações focadas em educação patrimonial junto a escolas de ensino fundamental e médio, projetos acadêmicos no âmbito da formação dos alunos da própria UNICAMP, divulgação científica por meio de revista arbitrada (Revista Arqueologia Pública) e, agora, debates em fóruns e redes específicos no tema da musealização da arqueologia e acervos arqueológicos. Um ponto comum em todas as ações institucionais está na premissa do laboratório como um espaço aberto para o encontro dos alunos, formação de pesquisadores e local de realização de pesquisas. Não é demérito afirmar que, como instituição, estamos longe de ocupar o espaço que desenhamos para nós: imaginamos o LAP como um palco para a realização de rodas de conversas e debates que se iniciem no campo da arqueologia, mas que atinjam questões do próprio bem-viver (SANTOS, 2010) ${ }^{5}$. Desejamos um laboratório que possa acolher e ao mesmo tempo ser acolhido, sempre a partir do diálogo aberto e democrático com as comunidades plurais às quais ele pertence.

Tendo como cenário nossa trajetória passada, nosso presente e nossos desenhos de futuro, nossa proposta neste texto é fazer um balanço de nossas ações e algumas propostas para o nosso presente mais imediato. Com esse objetivo, podemos afirmar que ao longo dos anos o LAP formou e foi formado por inúmeros pesquisadores das mais diferentes áreas, o que reafirmou a pluralidade de práticas arqueológicas e diálogos públicos. As pesquisas que temos em nosso histórico variam desde a arqueologia subaquática, processos de romanização na europa, estudos de gêneros, patrimônios ambientais e culturais, etnoarqueologia e arqueologia comunitária. Apesar da variedade temática, teórica e metodológica, a reunião dessas pesquisas (e pesquisadores) visava à discussão e à construção conceitual e prática de um campo comumente denominado como Arqueologia Pública (CARVALHO; FUNARI, 2009) ${ }^{6}$. Ao lidar com o controverso tema da arqueologia por contrato no cerne de nossa própria existência, fomos expostos à necessidade de ação da reflexão e ação política de forma ativa. Os caminhos não foram e não são fáceis. Nosso acervo está em constante mudança, não só no seu aspecto organizacional como também na forma de relação que se estabelece com o acervo, especialmente refletido nos projetos desenvolvidos pelo LAP. Ou seja, o acervo está vivo e em movimento. E esse é o ponto que gostaríamos de destacar: é possível pensar em um acervo que seja cientificamente catalogado dentro das normas estabelecidas pela ciência

\footnotetext{
${ }^{5}$ SANTOS, Boaventura de Sousa. Refundación del Estado en América Latina: perspectivas desde una epistemologia Del Sur. Quito: AbyaYala, 2010.

${ }^{6}$ CARVALHO, A. V.; FUNARI, P. P. A. Arqueologia Pública: as relações entre a academia e a sociedade. História e-História, v. 1, p. 1-15, 2009.

(C) Rev. Arqueologia Pública Campinas, SP \begin{tabular}{l|l|l} 
v.11 & n.2
\end{tabular} p. 255 Novembro/2017 1 ISSN 2237-8294
} 


\title{
Revista de Arqueologia Pública
}

arqueológica e seus órgãos institucionais, como também é possível pensar no acervo como um ente vivo que está em movimento e atua na formação plural e política de futuros profissionais e com sentido para as comunidades (ROBERTS, 1995) ${ }^{7}$.

\section{ALGUNS TEMAS CENTRAIS SOBRE A RELAÇÃO DE ARQUEOLOGIA PÚBLICA E ACERVOS}

O debate trazido pela normativa 196/2016 do IPHAN trouxe à tona reflexões que eram feitas em várias instituições universitárias, em diferentes momentos de inflexão. Seja por conta de adequações de espaços físicos, seja por respostas a demandas comerciais ou preocupações preservacionistas. No entanto, vale lembrar que esse debate, no cenário internacional, teve alguns pontos de reflexões que merecem um olhar aprofundado, especialmente quando falamos sobre Arqueologia Pública.

\section{NEUTRALIDADE CIENTÍFICA DOS ACERVOS}

É interessante notar como esse debate é incipiente quando se trata de acervos no Brasil, de certa forma compreensível já que a questão estrutural de um acervo tem sido o espaço de luta interna entre diversas instituições públicas, especialmente em tempos de crises e de corte financeiro para a manutenção desses espaços. No entanto, o pressuposto da neutralidade implica na coleta obsessiva de dados e amostras (ou todo material possível como se não houvesse amanhã, descartando a possibilidade de uma nova escavação, por gerações futuras) para se reconstruir a "verdadeira história" contada por um sítio arqueológico, deixamos de ter em perspectiva que um arquivo é montado com uma visão particular de história por uma pessoa em particular, como bem aponta Hedley Swain (2012), ainda que pautado no contexto britânico:

\begin{abstract}
The concept of preservation by record has also led to a philosophical dogma that there is a imperative that the archive should be a complete and objective record of an excavation that must be then kept forever as a record of what was once there. This search for "completeness" and "objective truth" also underlies much of current archaeological and museum-archaeological theory. It is perhaps now in need of revision. Much archaeological effort and practice in the last thirty years has been put into creating this objective mirror, in record form, of the original site and the "slice of history" that was revealed by its excavation. However, all archaeological interpretation is
\end{abstract}

\footnotetext{
${ }^{7}$ ROBERTS, Roda P. Community Interpreting Today and Tomorrow. In: CARR, S. E. The critical link: Interpreters in the community. Amsterdam: John Benjamins Publishing, 1997.
} 
subjective, open to different interpretations, and still heavily reliant on the individual perceptions of particular excavator, team of archaeologists, or site director. [...] There therefore needs to be a far more explicit acceptance that archaeological archives are not objective dispassionate records, but the particular interpretation of particular person. In addition there needs to be a more general acceptance that archaeological strada offer very partial windows into very particular histories. (2012, p. 352-353).

Esse é inclusive um dos motivos de ações de uma curadoria in situ, de se levar o "arquivo" mais próximo possível dos sítios em uma coleta sistematizada, com orientações científicas, museológicas e interpretativas de história bem determinadas. Sendo assim, não apenas uma coleta ampla e irrestrita de um "pedaço de um bolo de história", pautada em um sentido preservacionista com origens na pressuposta neutralidade científica.

\title{
COMMODITIES
}

Um dos conceitos que "salta aos olhos" neste debate, ainda que polêmico, mas com um forte sentido metafórico é arqueologia como commoditie, elaborado por Moshenka (2009) e pelos subsequentes artigos de réplica e tréplica de Gestrich (2011) e Moshenka \& Burtenshaw (2011):

\begin{abstract}
What is Public Archaeology? Introduced a model for studying archaeological economics based on the idea of archaeological commodities. In this reductionist model, archaeology is the process of the production of archaeological commodities, while public archaeology is the study and critique of the processes of production and consumption of the same. This interest in production and consumption is not, as Gestrich suggests, a "supply-and-demand model". In fact, one of the fundamental problems in public archaeology is the apparent disconnect between the producers and consumers of archaeology: the former often ignorant or uninterested in the demand; the latter just as often unaware of the supply. By some standards, a functioning supply-and-demand model might represent a substantial step forward. (MOSHENKA \& BURTENSHAW, 2011, p. 1).
\end{abstract}

Assim, vale ressaltar que essa percepção de commoditie é baseada na relações sociológicas marxistas de valores simbólicos e processos sociais contidos nas dinâmicas de produção de conhecimento arqueológico e de como ela é valorada pelo público em geral, arqueólogos especializados, entre outros grupos de interesse - stakeholders. Quantas vezes já não deparamos com frases como: "Meu acervo tem 40.000 peças!" e colocações similares? É possível que estas colocações, ainda que ditas em contextos informais, sejam esboços justamente de relações de commodities (e seu capital simbólico), posto por Moshenka (2009). O intrigante é que essa perspectiva suscita um aspecto de controle da commoditie, de controle da informação nos bancos de dados dos acervos arqueológicos.

\begin{tabular}{l|l|l} 
v.11 & n.2
\end{tabular}
p. 257 Novembro/2017 ISSN 2237-8294 


\title{
ARTIGO
}

\section{Revista de Arqueologia Pública}

Claro que o controle científico é extremamente necessário (especialmente em tempos de arqueologia por contrato), mas o acesso a essas informações, ponto nevrálgico, que deve ser posto à tona (lembremos que até hoje não são expostas as coordenadas de GPS no Cadastro Nacional de Sítios Arqueológicos CNSA/SGPA do IPHAN).

\section{HERANÇA CULTURAL}

O interesse geral (midiático, institucional, mercadológico etc.) pela arqueologia também é um dos campos cobertos pela Arqueologia Pública. Esta perspectiva é atrelada às percepções de valores entorno da herança cultural (heritage) nos seus diferentes aspectos, mas tendo como cerne os valores mobilizados e seus agentes no entorno dessa categorização, como bem aponta Michael Shanks (2005):

\begin{abstract}
This debate around heritage is about values, the crucial issue in public archaeology. The conservation ethic is driven by value being attached to the remains of the past. Should this or that site be conserved? Is this more worthy of display than that? And-in the antiquities markets, legal or illicit how much is this sculpture worth? All these questions of value are about what the past means to different people now. (2005, p. 166).
\end{abstract}

Assim, uma política de gestão de acervos ou mesmo seu sistema de informação organizacional (por mais técnico que seja) traz em si essa temática, e muitas vezes "esquecemos" de explicitar os valores relacionados a tal prática. A explicitação de tais valores e seus debates não só deveriam ser encaradas como uma importante atitude metodológica, como também uma rica fonte de conhecimento sobre a epistemologia da ciência arqueológica. Este é um dos cernes da Arqueologia Pública, inclusive, neste sentido encontramos também a importância de se criar arquivos historiográficos daqueles que foram responsáveis pela criação dos arquivos arqueológicos.

\section{ACESSIBILIDADE}

Uma das conquistas recentes que aos poucos vêm sendo implementada pelas instituições públicas é a lei da a Lei Complementar 131, também conhecida como Lei da Transparência, sancionada em 2009. O acesso ao dado público, reverbera (ou ao menos deveria) na forma como que é organizado o sistema de informação que gerencia o acervo arqueológico de uma instituição. Não basta o dado estar aberto a consulta pública, mas estar de fato acessível e organizado. Pode parecer uma obviedade escrever tais palavras, no entanto, a dificuldade de acesso aos acervos no Brasil a fora não é pouca e de fato dificulta a interpretação de dados arqueológicos, especialmente em estudos comparativos. Um dos pilares da Arqueologia Pública é justamente essa acessibilidade, não apenas para

\begin{tabular}{l|l|l|} 
v.11 & n.2 & p. 258
\end{tabular}


uma questão de acesso e interpretação ao registro arqueológico, mas também para a compreensão da formação das próprias coleções, de uma historiografia intelectual da atividade arqueológica. Importante apontar nesse contexto que a digitalização de acervos e documentos é uma peça central desse processo, que não deve ser feita sem uma perspectiva de metadados bem orientadas justamente para não enfraquecer os aspectos de acessibilidade, como bem aponta Jeffrey et al. :

In recent years, the detrimental effect of inaccessibility and difficulty of discovery of the large amounts of archaeological information represented by this material has begun to be recognized by the academic community. Prominent researchers, such as Bradley (2006) and Lock (2008), have questioned why it is not more widely available. Digital hosting and online delivery of this material, both of newly created material (i.e. "born digital") and digitized versions of legacy documents, would seem a logical approach to addressing these access issues. However, good access is predicated on good discovery mechanisms and these rely, among other things, on good metadata (JEFFREY et al., 2009).

\section{O CONCEITO DE ARQUEOLOGIA PÚBLICA EM UMA PRÁTICA LABORATORIAL}

À primeira vista, poderia-se pensar em uma certa contradição das propostas de atuação do LAP, já que seu acervo é composto majoritariamente por peças advindas da "arqueologia de contrato ou preventiva", no entanto, tal situação é nossa reflexão de entrada sobre política arqueológica e história arqueológica do Brasil. Muitas vezes, não basta uma disciplina acadêmica para compreender como esses processos funcionam, mas vivenciar um acervo que foi construído nestes processos, nesta história de grandes desenvolvimentos e contradições da arqueologia contemporânea brasileira potencializada pela Normativa IPHAN de 2003. O boom de acervos, como sabemos, não foi por acaso.

Como outras instituições de guarda de acervos arqueológicos, a reserva técnica (RT) foi se intensificando com o acúmulo material e documental, chegando ao ponto de uma reformulação e readequação do espaço físico do laboratório. A fundação do LAP foi arquitetada na proposta da Arqueologia Pública, como já explicado anteriormente. Assim, nosso desafio teórico, metodológico e prático estava em como associar duas vertentes (Gestão de Acervos e Arqueologia Pública) que pouco dialogavam até aquele momento. Como primeiro passo, construímos a proposta de tornar público e de fácil localização os documentos associados aos projetos contidos na RT e explicitar geograficamente o material arqueológico. Essa cartografia exposta de nosso acervo parecia uma estratégia interessante para reafirmar a dinâmica viva do material arqueológico contido na nossa reserva, pôr-lhe em movimento tanto em termos educacionais quanto em termos reflexivos acadêmicos. No que tange à publicização documental, escolhemos utilizar a plataforma do 
Google Drive para a organização documental interna já com o esboço de publicização final. Alinhada a esse processo está a criação do mapa ilustrativo, também na plataforma do Google Maps. Acerca dos termos acadêmicos, está no alinhamento da Revista de Arqueologia Pública trabalhar com o acervo contido no próprio laboratório, seja na escolha de artigos relacionados ao material, seja na futura obrigação de entrega de um artigo científico junto ao recebimento de material arqueológico advindos de endossos institucionais.

Por fim, cabe-nos refletir sobre como é possível enquadrar preceitos da Arqueologia Pública junto a normas cristalizadas pelo ente público, especialmente dadas em um sentido de diálogo verticalizado (diálogos horizontais nunca foram pressupostos do Estado brasileiro). Assim, situações que poderiam ser contempladas com práticas laboratoriais são justamente aquelas que tratam de comunidades tradicionais, de criar sentidos de ressonância patrimonial que não se resumem na vida de laboratório de um objeto, reconhecendo, portanto, saberes tradicionais e privilegiando as diferentes visões de mundo $(\text { GNECCO, 2010 })^{8}$. Seria possível almejar essa perspectiva em uma prática laboratorial científica?

Há muito a ser feito, principalmente no que se refere às múltiplas apropriações de nosso espaço e acervo. Por isso, estamos aqui. Como construir caminhos para a democratização de nossas ações, espaços e acervo? Em um momento de tanta fragilidade da política democrática, parece-nos necessário defender a multivocalidade ${ }^{9}$ em amplos e diferentes níveis. O texto que segue abaixo faz parte de nosso protocolo de ação em relação ao acervo. Sua premissa é a defesa da integridade física do patrimônio arqueológico, mas também de toda rede de conhecimento que pode estar a ele atrelado .

\section{Conclusão}

\section{Sistematizando um protocolo com a inclusão "pública"}

Postas as reflexões acima e as correlações possíveis sobre a Arqueologia Pública, transcrevemos aqui um fragmento do nosso protocolo de recebimento de material arqueológico, para que ilustremos um pouco que é sim possível aplicar uma série de valores atrelados a percepções de herança cultural em um protocolo:

Considerando as instruções do IPHAN sobre o patrimônio arqueológico: Portaria do IPHAN n. 07 de 1988, Instrução Normativa n. 01 de 2015 e as recomendações que

\footnotetext{
${ }^{8}$ GNECCO, Cristobal. Da Arqueologia do Passado à Arqueologia do Futuro: Anotações sobre Multivocalidade e Multiculturalismo. Colômbia: Ed. Universidade de Calcutá, 2010.

${ }^{9}$ MERRIMAN, Nick (Org). Public Archaelogy. London: Ed. Routledge, 2004.

\begin{tabular}{|l|l|l|l|l|l|l|}
\hline (C) Rev. Arqueologia Pública & Campinas, SP & v.11 & n.2 & p. 260 & Novembro/2017 & ISSN 2237-8294
\end{tabular}
} 


\section{ARTIGO}

\section{Revista de Arqueologia Pública}

constam na Portaria do IPHAN n. 196 de 2016, o Laboratório de Arqueologia Pública Paulo Duarte (LAP), da Universidade Estadual de Campinas (UNICAMP), indica abaixo os procedimentos que estamos regulamentando para nossa rotina. A aceitação do Endosso Institucional do LAP para a guarda do material arqueológico implica necessariamente na concordância das seguintes normas:

\section{Documentação:}

Toda a documentação original produzida em campo e em laboratório - diários de campo, fichas de análises, registros audiovisuais, desenhos, plantas, fotos, fichas topográficas, croquis, fichas de catálogo e inventário - bem como os relatórios parciais e o relatório final deverão ser entregues DIGITALIZADOS junto ao material arqueológico. Não será aceita a entrega de material arqueológico sem o acompanhamento dessa documentação. Atenção para os formatos das planilhas de inventário, que devem ser entregues no formato excel, e as resoluções das fotos, que devem ser no mínimo de 300 dpi.

A documentação deve ser separada por sítio, inclusive no diário de campo, salvo pesquisas que tenham somente a presença de áreas de ocorrências ou procedimentos de prospecções gerais.

Devem ser entregues, em documentos digitalizados específicos, os seguintes documentos:

* Formulário de Sítio Arqueológico do Sistema de Gestão do Patrimônio Arqueológico (atualizado) do Cadastro Nacional de Sítios Arqueológicos (IPHAN), em Access

* Inventário de material arqueológico em planilha excel nos moldes dos itens classificatórios estabelecidas pela "Fichas de Cadastro de Bem Arqueológico Móvel" (Portaria IPHAN 196/2016), acrescido de algumas informações (data; invólucro; caixa; nível/quadra/prospecção; coord UTM), vide tabela exemplo:

\begin{tabular}{|c|c|}
\hline Sítio & SLL \\
\hline Data & $22 / 02 / 2017$ \\
\hline Caixa & 5 \\
\hline Involucro & 2 \\
\hline Coord UTM & 22681462 \\
& 47556535 \\
\hline Nivel & 3 \\
\hline Quadra & A1 \\
\hline Categoria & Artefato \\
\hline Sub-categoria & fragmento \\
\hline Material & cerâmica \\
\hline Cor & policromático \\
\hline Técnica de Produção & modelado \\
\hline NP & 102 \\
\hline
\end{tabular}




\section{ARTIGO}

\section{Revista de Arqueologia Pública}

* Listagem de Caixas, em que deve constar o nome do Sítio/AOA, categorias materiais/especificidades, total de invólucros e total de peças por caixa

* Listagem das Fotografias realizadas em campo, em laboratório, em atividades educativas, entre outras: identificação das pastas, subpastas (se houver) e das fotos; local; autoria; data; observação (se houver). Informar as legendas das identificações escolhidas

* Fichas de Cadastro de Bem Arqueológico Móvel (Portaria IPHAN 196/2016, Anexo II). Esta Ficha é utilizada para compor o Termo de Recebimento de Acervo Arqueológico (segundo Portaria 196, 18/05/16, Anexo III, IPHAN)

* Arquivo digital com as coordenadas dos sítios no formato do Google Earth (.kml).

\section{ARQUEOLOGIA PÚBLICA, DEMANDAS ESPECÍFICAS}

No âmbito da especificidade do trabalho do LAP, solicitamos obrigatoriamente a apresentação de:

* Uma proposta de trabalho de educação patrimonial ou de Arqueologia Pública atrelada ao material arqueológico entregue. Proposta esta que pode ser feita no formato de projeto acadêmico para um futuro aluno ou estagiário do LAP

* Um artigo científico relacionado ao material entregue para ser publicado no âmbito da Revista de Arqueologia Pública (Qualis B1). Este artigo terá sua autoria respeitada e passará pelos trâmites normais da revista para a aprovação e futura publicação.

\section{REFERÊNCIAS BIBLIOGRÁFICAS}

BRADLEY, R. Bridging the two cultures. Commercial archaeology and the study of prehistoric Britain. Antiq. J. 86, 1-13, 2006.

CARVALHO, A. V.; FUNARI, P. P. A. Arqueologia Pública: as relações entre a academia e a sociedade. História e-História, v. 1, p. 1-15, 2009.

FREIRE, Paulo. Educação como prática da liberdade. 23르 ed. Rio de Janeiro: Paz e Terra, 1999.

GESTRICH, Nikolas. Putting a Price on the Past: The Ethics and Economics of Archaeology in the Marketplace - A Reply to "What is Public Archaeology". Present Pasts, v. 3, n. 2, p. 81-82, 2011.

GNECCO, Cristobal. Da Arqueologia do Passado à Arqueologia do Futuro: Anotações sobre Multivocalidade e Multiculturalismo. Colômbia: Ed. Universidade de Calcutá, 2010.

GONZÁLEZ-RUIBAL, A.; HERNANDO, A.; POLITIS, G. Ontology of the self and material culture: arrow making among the Awáhunter-gatherers (Brazil). Journal of Anthropological Archaeology, n. 30, p. 1-16, 2011.

MERRIMAN, Nick (Org). Public Archaelogy. London: Ed. Routledge, 2004.

\begin{tabular}{|l|l|l|l|l|l|l|} 
(C) Rev. Arqueologia Pública & Campinas, SP & v.11 & n.2 & p. 262 & Novembro/2017 & ISSN 2237-8294
\end{tabular}


MOSHENSKA, G. What is Public Archaeology?. Present Pasts, v. 1, n. 1, p. 46-48, 2010.

MOSHENSKA, G.; BURTENSHAW, P. Response: the Values of Archaeology. Present Pasts, v. 1, n. 1, p. 55-56, 2010.

ROBERTS, Roda P. Community Interpreting Today and Tomorrow. In: CARR, S. E. The critical link: Interpreters in the community. Amsterdam: John Benjamins Publishing, 1997.

SANTOS, Boaventura de Sousa. Refundación del Estado en América Latina: perspectivas desde una epistemologia Del Sur. Quito: AbyaYala, 2010.

SHANKS, Michael. PUBLIC ARCHAEOLOGY/MUSEOLOGY/CONSERVATION/HERITAGE. In: RENFREW, Collin; BAHN, Paul. Archaeology: key concepts. Routledged. London. 2005.

SWAIN, H. Archive Archaeology. In: The Oxford Handbook of Public Archaeology. Oxford University Press. 2012

JEFFREY, S. et al. The Archaeotools project: faceted classification and natural language processing in an archaeological context. Phil. Trans. R. Soc. A, v. 367, p. 2507-2519, 2009. 